\title{
Comparing nutritional requirements, provision and intakes among patients prescribed therapeutic diets in hospital: an observational study
}

\section{Author information:}

1. Megan Rattray, MNutrDiet, School of Allied Health Sciences, Griffith University, Gold Coast Campus, QLD 4222 Australia.

Email: megan.rattray@griffithuni.edu.au

2. Ben Desbrow, PhD, School of Allied Health Sciences; and Menzies Health Institute Queensland, Griffith University, Gold Coast Campus, QLD 4222 Australia. Email: b.desbrow@griffith.edu.au

3. Shelley Roberts, PhD, NHMRC Centre of Research Excellence in Nursing; and Menzies Health Institute Queensland, Griffith University, Gold Coast Campus, 4222 Australia. Email: s.roberts@griffith.edu.au

Author contributions: All authors contributed to study design and conception; MR contributed to data collection; and all authors contributed to data analysis and interpretation; and drafting, reviewing and approving the final version of the manuscript.

\section{Corresponding Author:}

Megan Rattray

Address: $\quad$ School of Allied Health Sciences, Griffith University, Gold Coast

Campus, QLD 4222 Australia

Email: $\quad$ megan.rattray@griffithuni.edu.au

Alternative email: m.rattray@griffith.edu.au

Tel number: +61756780154 


\begin{abstract}
Objective: Nutrition is an important part of patients' recovery in hospital. This study assessed the nutritional adequacy of meals provided to and consumed by patients prescribed a therapeutic diet.
\end{abstract}

Research: Methods \& Procedures: Patients $(n=110)$ prescribed a therapeutic diet (texture modified, low fibre, oral fluid or food allergy/intolerance diets) for medical or nutritional reasons were recruited from six wards of a tertiary hospital. Complete (24hr) dietary provisions and intakes were directly observed and analysed for energy $(\mathrm{kJ})$ and protein $(\mathrm{g})$ content. A chart audit gathered demographic, clinical and nutrition-related information to calculate each patient's disease-specific estimated energy and protein requirements. Provisions and intakes were considered adequate if they met $\geq 75 \%$ of patients' estimated requirements.

Results: Mean energy and protein provided to patients $(5844 \pm 2319 \mathrm{~kJ}, 53 \pm 30 \mathrm{~g})$ were significantly lower than their mean estimated requirements $(8786 \pm 1641 \mathrm{~kJ}, 86 \pm 18 \mathrm{~g})$. Consequently, mean nutrition intakes $(4088 \pm 2423 \mathrm{~kJ}, 37 \pm 28 \mathrm{~g})$ were significantly lower than estimated requirements. Only $37 \%(n=41)$ of patients were provided with and $18 \%(n=20)$ consumed adequate nutrition to meet their estimated requirements. No therapeutic diet provided adequate food to meet the energy and protein requirements of all recipients. Patients on oral fluid diets had the highest estimated requirements $(9497 \pm 1455 \mathrm{~kJ}, 93 \pm 16 \mathrm{~g})$ and the lowest nutrient provision (3497 $\pm 1388 \mathrm{~kJ}, 25 \pm 19 \mathrm{~g})$ and intake $(2156 \pm 1394 \mathrm{~kJ}, 14 \pm 14 \mathrm{~g})$.

Conclusion: Hospitalised patients prescribed therapeutic diets (particularly fluid only diets) are at risk of malnutrition. Further research is required to determine the most effective strategies to improve nutritional provision and intake among patients prescribed therapeutic diets.

\title{
Keywords
}

Adequacy; malnutrition; oral fluids; specialised diets 


\section{Introduction}

Protein-energy malnutrition (PEM) has profound consequences for patients and hospitals [1-5]. Undernourished patients are at increased risk of infections [3], falls [2] pressure injuries [1], and morbidity and mortality [4]. For hospitals, PEM results in increased costs associated with longer lengths of stay, higher readmission rates and greater resource utilisation [4, 5]. While the aetiology of PEM is multifactorial [6], attention is commonly drawn to inadequate dietary intake as the major modifiable cause [7-10]. Despite this, poor food intake remains problematic among inpatients [7-9, 11-15] and PEM affects 20-50\% of patients worldwide $[11,16,17]$. In order to develop specific interventions to address PEM and its associated consequences, a deeper understanding of why hospitalised patients eat inadequately is required.

Limited dietary selections, increased nutritional needs and/or decreased appetite may place some patients at greater risk of inadequate nutritional intake than others [18]. Patients on therapeutic diets may be particularly vulnerable to these factors, considering such diets are restrictive by nature (e.g. low gluten or low lactose diets for allergies/intolerances) [19] and commonly prescribed during times of acute illness when requirements are higher (e.g. oral fluid or low fibre diets for perioperative/medical care) [18]. Several studies have reported a higher proportion of patients prescribed therapeutic diets eat poorly compared to those on regular diets $[8,10,20,21]$. However, these studies have either a) involved small sample sizes, drawn from studies involving total hospital populations where only a limited proportion of patients have received therapeutic diets $(15-17 \%)[8,10]$ or b) investigated a small group of therapeutic diets (e.g. texture modified diets only [21]), limiting the generalisability of these findings to patients on all therapeutic diets. Given these constraints, our current understanding of the impact being prescribed a therapeutic diet plays in the potential to develop PEM is poor.

The purpose of this study was to assess the nutritional adequacy of meals (in respect to individually estimated requirements) provided to and consumed by patients prescribed a therapeutic diet in an acute care environment. Increased understanding of food provision and intake among this potentially vulnerable patient population may help inform strategies and resource allocation to prevent/treat malnutrition. 


\section{Material and Methods}

\section{Study overview}

This observational study assessed the nutritional adequacy of therapeutic diets provided to and consumed by patients. The study was approved by the relevant hospital and university Human Research Ethics Committees (reference numbers: HREC/13/QGC/162 and AHS/24/14/HREC).

\section{Setting}

Data was collected on six wards (gastrointestinal, short stay surgical, surgical, neurological, neurovascular, and rehabilitation) in a large metropolitan teaching hospital in Southeast Queensland. An electronic foodservice system (EFS; Delegate Software, Australia) was in operation whereby staff entered patients' dietary prescriptions into the system and patients ordered their main meals in accordance with their diet code via a bedside patient entertainment system screen ( $\geq 2 \mathrm{hrs}$ in advance of meal delivery). Electronic orders were sent to the kitchen and individual meal tickets were printed for meal assembly. Main meals were plated cold and heated or kept cool in temperature-controlled delivery trolleys (Burlodge, Australia) at the ward level. The EFS was not in operation for mid-meals, instead patients choose their mid-meals at bedside point-of-service

\section{Participants}

Patients were eligible to participate if they were: (a) able to provide informed consent (aged $\geq 18$ years, cognitively intact, and able to communicate in English); (b) prescribed one of the following therapeutic diets: (1) clear fluids (2), free fluids, (3) low fibre, (4) low lactose, (5) low gluten, (6) low allergen, (7) soft, (8) minced and moist, (9) smooth pureed, (10) mildly thick, (11) moderately thick, and (12) extremely thick; and (c) an inpatient for $\geq 24$ hours prior to the commencement of data collection, allowing sufficient time for staff to enter the patient's diet prescription into the EFS. Patients were excluded if they were: (a) palliative or dying; (b) unable to provide informed consent; or (c) previously enrolled in the study, to avoid duplication of data collection. Given the exploratory nature of this study, a sample size was not predetermined. Rather, a pragmatic approach was adopted, by which wards were approached in a random order until recruitment targets were reached $(n=8 /$ day; the number of patients for which data were able to be collected by the one researcher). 


\section{Data collection}

A schedule was developed to ensure each day of the hospital's 14-day cyclic menu was observed on two separate occasions (i.e. totalling 28 days of data collection) over a 6-week period (February - March 2015). The sequencing of these days were randomised via a computer generated roster. A chart audit was conducted using the patient's electronic medical record and bedside chart to obtain demographic (age, gender, diagnosis, comorbidities and length of stay) and nutrition-related information (weight, height and prescribed diet).

The nutritional adequacy of food provided to and consumed by patients was determined by a) recording each patient's dietary provision and intake over a 24 hour period, b) calculating their total energy and protein provision and intake, and c) comparing these values to their individually estimated energy and protein requirements. Patients' nutrition provision and intakes were considered adequate if they met $\geq 75 \%$ of their estimated requirements, which has previously been shown to be sufficient for weight maintenance among inpatients [13]. Patients' disease-specific estimated energy requirements (EER) and estimated protein requirements (EPR) were calculated using Australian best practice clinical guidelines for patients with specific disease states, and 100-125kJ/kg (25- 30kcal/kg) and 0.8-1.0g/kg protein for individuals without disease states affecting metabolic requirements[22].

Visual observation was used to quantify patients' dietary intake for all main meals (i.e. breakfast, lunch, dinner). Consumption of each dietary item delivered was recorded as a fraction of the whole portion on a five-point scale (none, $1 / 4,1 / 2,3 / 4$, all), which has previously been shown to correlate closely with weighed dietary intake [15, 23, 24]. If patients kept dietary items for later consumption, it was noted and the intake of the item/s was recorded at a later time (usually the next main meal). Mid-meal (morning tea, afternoon tea and supper) dietary items provided by the hospital, including oral nutrition supplements (ONS) and any personal dietary items (i.e. purchased at cafeterias or vending machines, or brought in by family or friends) were estimated by visual observation or patient recall and recorded, to capture a complete 24hr dietary intake. One researcher independently collected all observational and chart audit data to eliminate inter-rater variability.

Complete 24hr food intake was not able to be collected for all participants. Patients were excluded from analysis if over the 24hrs of observation they: (a) were instructed nil by mouth for a period of the day; (b) had their diet upgraded to a code not being investigated (e.g. 
full/unrestricted diet); (c) were moved to another ward or were discharged before they reached 24hrs of dietary observation; or (d) were prescribed enteral or parenteral feeding in conjunction with dietary provision.

\section{Data analysis}

All data were entered into SPSS version 22.0 for Windows (IBM Corp. 2012, Armonk, N.Y., USA). Following data entry, a random data check was completed on $10 \%$ of the data to assess for entry errors. This check yielded $<1 \%$ errors.

All observational dietary data was entered into a Foodworks database (Xyris software, Australia) which contained the energy (kilojoule, kJ) and protein (grams, g) composition of all dietary items provided by the hospital for each therapeutic diet investigated. To calculate the total energy and protein provided to each patient, all dietary items delivered at main and mid-meals by the hospital (including oral nutrition supplements) were entered into Foodworks as whole portions. To determine the total energy and protein consumed by each patient, their intake of each dietary item at main and mid-meals was entered into the Foodworks database as a fraction of consumption. Any personal dietary items consumed by patients were excluded from provision analysis, but included in intake analysis.

Diet types were grouped into four categories for analysis: texture modified diets (TMD) (all TMD foods and thickened fluids), oral fluids (clear and free fluids), food allergy/intolerance diets (low allergy, lactose and gluten) and low fibre diets. The proportion of patients provided with and having consumed adequate energy and protein (in relation to their individual EER and EPR) were categorised into binary variables (i.e. consumed $\geq 75 \%$ or $<75 \%$ of requirements) and reported as number and percentage of patients who met their EER and EPR.

Descriptive statistics were used to outline patient demographics, admission characteristics and nutrition related information. Independent t-tests, Mann-Whitney U Tests or Chi-square tests were used to determine any differences between demographics of patients included and not included (incomplete 24hr intake and provision data) in the analysis. Wilcoxon Singed Rank Tests were used to analyse the variation in mean energy and protein requirements, provision and intake among all patients. Mann-Whitney $U$ tests were run to analyse the 
variation between diet prescriptions on patients' estimated energy and protein requirements, intakes and provision. For all associations, significance was set at $\mathrm{p} \leq 0.05$.

\section{Results}

\section{Patient characteristics}

Of 186 patients approached and informed of the study's protocol, 175 agreed to participate and complete data was obtained from 110 (see Figure 1). For 57 patients, complete dietary intake data was unavailable (i.e. patients were discharged or met study exclusion criteria prior to 24hrs of dietary intake data being collected). There were no significant differences in patient demographics between those included and excluded from the study. Patient demographics are described in Table 1. Patients' admission type (i.e. medical or surgical) and diagnoses are described in Table 2.
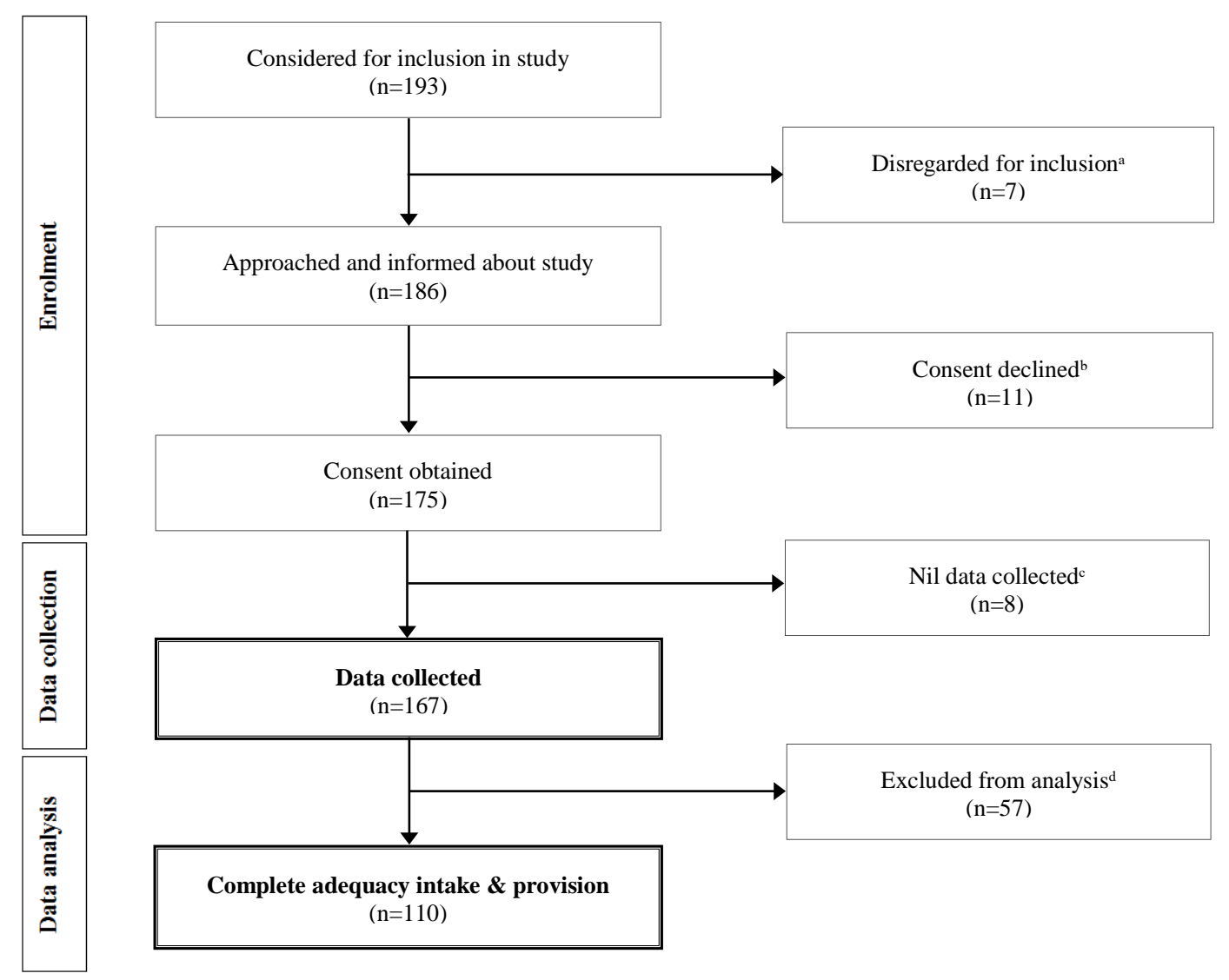

Figure 1 Patient recruitment and retention

a Patient met exclusion criteria

b Reasons included: patient too ill $(n=3)$; negative attitude towards foodservices $(n=2)$ or students $(n=2)$; or no reason given $(n=4)$

c Reasons included: patient discharged $(n=2)$; moved $(n=2)$; or had diet code changed $(n=4)$ before data collection initiated

d Patient did not fulfil inclusion criteria for adequacy analysis 
Table 1 Demographic characteristics of study patients $(n=110)$

\begin{tabular}{|c|c|}
\hline Demographic characteristic & Mean \pm SD \\
\hline Age (years) & $53.8 \pm 18.0$ \\
\hline Gender (female) ${ }^{a}$ & 61 (55.5\%) \\
\hline Length of stay (days) ${ }^{b}$ & $6.3(2-68)$ \\
\hline Weight (kg) & $77.2 \pm 19.2$ \\
\hline
\end{tabular}

[SD: Standard deviation]

${ }^{\text {a }}$ Presented as $\mathrm{n}(\%)$

${ }^{\mathrm{b}}$ Presented as median (IQR) presented due to non-normal distribution

Table 2 Admission characteristics of all recruited patients with complete provision and intake data

\begin{tabular}{lll}
\hline Admission characteristic & & Result n (\%) \\
\hline Admission type & & \\
& Medical & $60(55)$ \\
Main diagnostic categories & Surgical & $50(45)$ \\
& Gastrointestinal (medical and surgical) & $61(56)$ \\
& Neurological & $15(14)$ \\
& Reproductive & $6(5)$ \\
& Oncology (medical and surgical) & $5(4)$ \\
& Musculoskeletal & $5(4)$ \\
& Renal & $4(4)$ \\
& Respiratory & $2(2)$ \\
& Cardiovascular & $2(2)$ \\
& Immunological & $1(1)$ \\
Comorbidities & Others & $9(8)$ \\
& & \\
& & $15(14)$ \\
& No comorbidities & $47(43)$ \\
& Cardiovascular & $34(31)$ \\
& Gastrointestinal & $16(15)$ \\
& Respiratory & $17(16)$ \\
Musculoskeletal & $13(12)$ \\
Mental & $12(11)$ \\
& Diabetes & $3(3)$ \\
& Cancer & $23(21)$ \\
\hline & Renal &
\end{tabular}


Table 3 Estimated requirements, provision and intake of energy and protein for all participants $(\mathrm{n}=110)$ and among different diet types

\begin{tabular}{|c|c|c|c|c|c|c|}
\hline Estimated variable & & All $(n=110)$ & \multicolumn{4}{|c|}{ Diet type } \\
\hline Requirements & Protein (g) & $86 \pm 18$ & $85 \pm 16$ & $73 \pm 24$ & $93 \pm 16^{\mathrm{e}}$ & $78 \pm 17$ \\
\hline Provision & Protein (g) & $53 \pm 30$ & $71 \pm 21$ & $64 \pm 18$ & $25 \pm 19^{f}$ & $72 \pm 24$ \\
\hline \multirow{2}{*}{ Intake } & Energy (kJ) & $4088 \pm 2423$ & $5084 \pm 2029$ & $5471 \pm 2617$ & $2156 \pm 1394^{\mathrm{f}}$ & $5864 \pm 2163$ \\
\hline & Protein (g) & $37 \pm 28$ & $46 \pm 23^{g}$ & $62 \pm 31$ & $14 \pm 14^{\mathrm{f}}$ & $61 \pm 28$ \\
\hline
\end{tabular}

[Food A/I; Food allergy and intolerance; TMD: Texture modified diets]

a Soft, minced and moist, smooth pureed, mildly thick, moderately thick and extremely thick

${ }^{\mathrm{b}}$ Low allergen, gluten and lactose

c Clear and free fluids

d Signifies a significant difference $(\mathrm{p}<0.004)$ between oral fluids and TMD and low fibre (Mann-Whitney U)

e Signifies a significant difference $(\mathrm{p}<0.05$ ) between oral fluids and all other diet codes (Mann-Whitney U)

f Signifies a significant difference $(\mathrm{p}<0.001)$ between oral fluids and all other diet codes (Mann-Whitney U)

${ }^{g}$ Signifies a significant difference $(\mathrm{p}<0.001)$ between TMD and low fibre (Mann-Whitney U) 


\section{Adequacy of dietary intake and provision}

Mean energy and protein requirements, provision and intake for all patients and across all diet types are presented in Table 3. The total mean energy and protein provided to all patients $(5844 \pm 2319 \mathrm{~kJ}$ and $53 \pm 30 \mathrm{~g})$ were significantly lower than patients' mean EER $(8786 \pm 1641 \mathrm{~kJ})$ and EPR $(86 \pm 18 \mathrm{~g})(\mathrm{p}<0.001)$. Consequently, mean energy $(4088 \pm 2423 \mathrm{~kJ})$ and protein $(37 \pm 28 \mathrm{~g})$ intakes were significantly lower than estimated requirements $(\mathrm{p}<0.001)$ and provision $(\mathrm{p}<0.001)$. There were differences between diet codes in patients' mean energy and protein requirements, provision and intakes (Table 3). Patients on oral fluid diets had significantly higher mean estimated requirements and significantly lower nutrient provisions and intakes compared to patients on other diets. No significant differences in energy and protein intakes were observed among patients with a $\mathrm{BMI} \leq 25$ and those with a $\mathrm{BMI}>25$.

Only 41 (37\%) patients were provided with adequate food to meet both EER and EPR. Subsequently, only 20 (18\%) patients ate enough food to meet $\geq 75 \%$ of their EER and EPR. No therapeutic diet provided adequate food to meet all recipients' nutritional requirements (see Table 4). This was particularly evident among patients prescribed oral fluid diets.

Table 4 Number and proportion of patients provided with and consumed adequate food to meet estimated energy and protein requirements

\begin{tabular}{|c|c|c|c|c|c|c|}
\hline \multicolumn{2}{|c|}{ Estimated variable } & \multirow[t]{2}{*}{ All $(n=110)$} & \multicolumn{4}{|c|}{ Diet type } \\
\hline & & & $\begin{array}{l}\mathrm{TMD}^{\mathrm{a}} \\
(\mathrm{n}=43)\end{array}$ & $\begin{array}{c}\text { Food A/I }{ }^{b} \\
(n=6)\end{array}$ & $\begin{array}{l}\text { Oral fluids } \\
\quad(n=43)\end{array}$ & $\begin{array}{c}\text { Low fibre } \\
(\mathrm{n}=18)\end{array}$ \\
\hline \multirow{2}{*}{ Provision } & Energy & $55(50 \%)$ & 35 (80\%) & $3(50 \%)$ & $2(5 \%)$ & 15 (83\%) \\
\hline & Protein & $44(40 \%)$ & $26(60 \%)$ & $4(67 \%)$ & $1(2 \%)$ & $13(72 \%)$ \\
\hline \multirow{2}{*}{ Consumption } & Energy & $26(24 \%)$ & $13(30 \%)$ & $3(50 \%)$ & $1(2 \%)$ & $9(50 \%)$ \\
\hline & Protein & $24(22 \%)$ & $10(23 \%)$ & $3(50 \%)$ & $0(0 \%)$ & $11(61 \%)$ \\
\hline
\end{tabular}

[Food A/I; Food allergy and intolerance; TMD: Texture modified diets]

a Soft, minced and moist, smooth pureed, mildly thick, moderately thick and extremely thick

b Low allergen, gluten and lactose

c Clear and free fluids

The mean proportion of patients' energy and protein requirements met through dietary intake differed according to the therapeutic diet investigated (see Figures 2 and 3). On average, patients prescribed oral fluid diets ate poorly, meeting $<25 \%$ of EER and EPR. Alternatively, 
patients on food intolerance/allergy diets and low fibre diets typically met a higher proportion of their EER and EPR ( $75 \%$ of EER and EPR met).

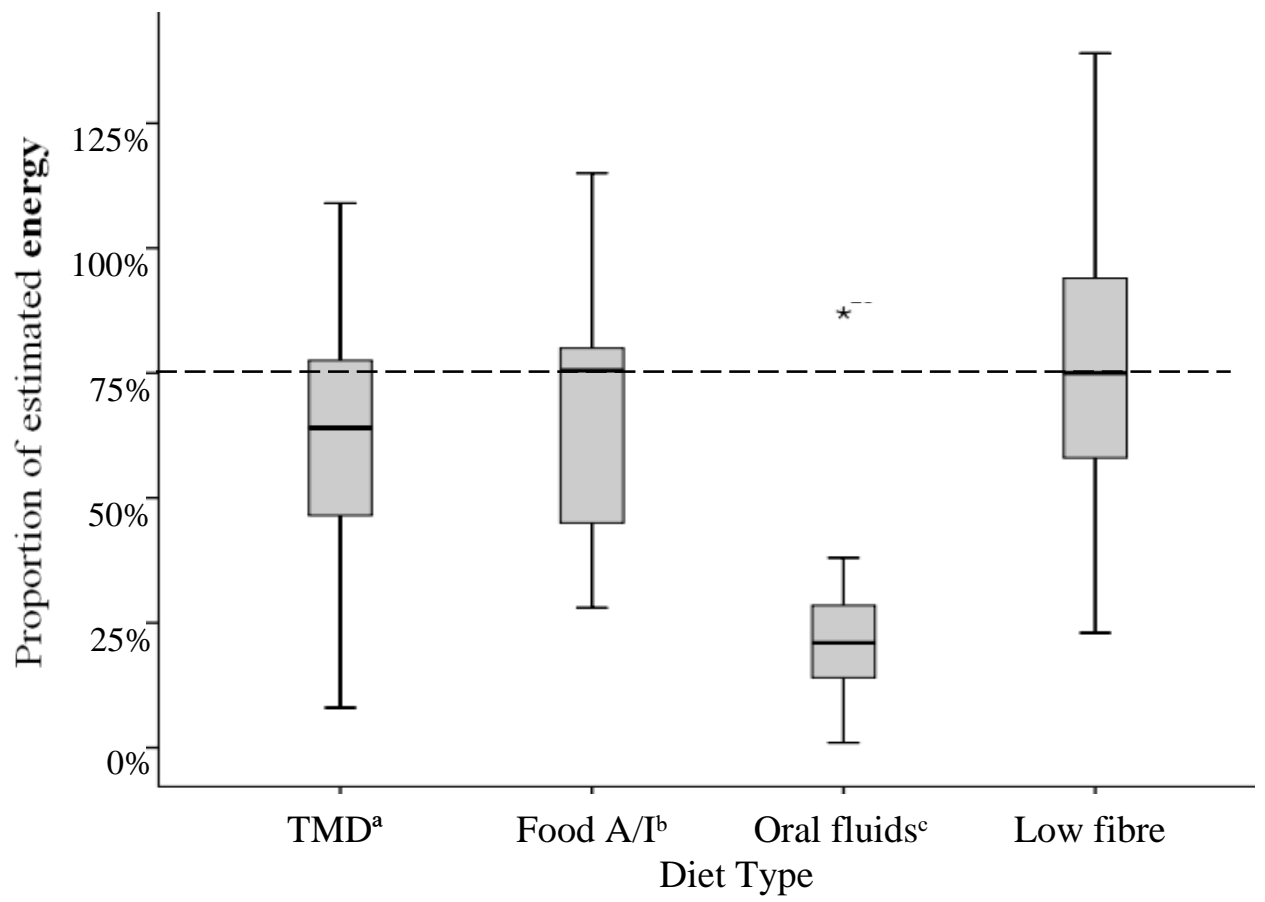

Figure 2 Mean proportion of energy needs met according to diet type

[Food A/I: Food allergy and intolerance; TMD: Texture modified diet]

Note: Dotted line illustrates estimated energy needs met ( $>75 \%$ of estimated requirements)

a Soft, minced and moist, smooth pureed, mildly thick, moderately thick and extremely thick

${ }^{\mathrm{b}}$ Low allergen, gluten and lactose

${ }^{c}$ Clear and free fluids

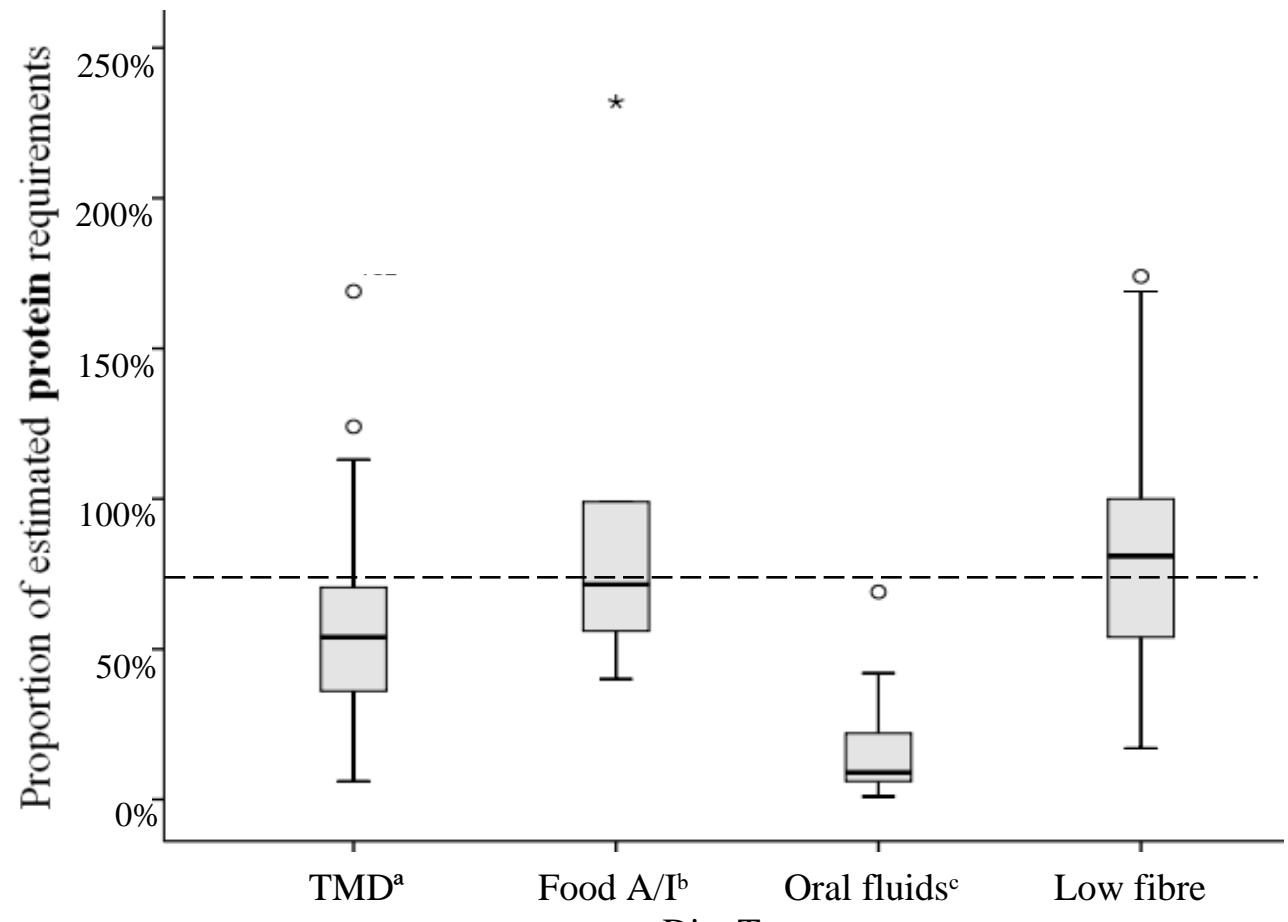

Diet Type

Figure 3 Mean proportion of protein needs met according to diet type

[Food A/I: Food allergy and intolerance; TMD: Texture modified diet]

Note: Dotted line illustrates estimated protein needs met ( $>75 \%$ of estimated requirements)

a Soft, minced and moist, smooth pureed, mildly thick, moderately thick and extremely thick

b Low allergen, gluten and lactose

c Clear and free fluids 
Forty (36\%) patients consumed personal dietary items during the 24hr of observation periods. For these patients, mean energy and protein intakes from personal dietary items were $1352 \pm 1205 \mathrm{~kJ}$ and $10.9 \pm 17.2 \mathrm{~g}$, contributing $27 \%$ and $22 \%$ to their total energy and protein intakes, respectively. In total, sixteen patients (15\%) received ONS over a $24 \mathrm{hr}$ period of observation. The prescription of ONS was most common among patients on oral fluid diets $(n=11,26 \%)$. 


\section{Discussion}

This study investigated the nutritional adequacy of meals delivered to and consumed by acute care patients prescribed therapeutic diets in hospital. Overall, energy and protein provision and intakes were poor, resulting in a minority (18\%) of patients meeting their estimated requirements. This may be attributed to a combination of factors, including: low nutrient provision (foodservice and nutritional care-related); high nutritional requirements (patientrelated); and reduced nutritional intake (patient and nutritional care-related). This suggests multifaceted strategies to improve nutritional intakes should be directed towards patients prescribed therapeutic diets, a highly vulnerable patient, to prevent/treat PEM.

The mean energy and protein provided to patients in the current study are lower than previously reported among inpatients $[8,10,15,25]$. This may be attributed to two main factors: 1) the exclusive inclusion of patients prescribed therapeutic diets and 2) the type of therapeutic diets investigated. By definition, a therapeutic diet eliminates one or more nutrients [19]. Any diet that eliminates certain nutrients/food groups poses a risk of nutritional inadequacy if nutrients/foods omitted are not sufficiently substituted [26, 27]. This was observed in the current study, with lower mean nutrition provisions seen across all therapeutic diets in comparison to previous studies investigating nutrition provision among patients on regular diets $[8,10,15,25]$. However, certain therapeutic diet types lend a greater risk to nutritional inadequacy than others. For example, patients on oral fluid diets (i.e. clear fluids or free fluids) were provided with approximately half the energy and a third of the protein compared to patients on TMD, food allergy/intolerance and low fibre diets. This most likely is due to the strict food choices permissible on oral fluid diets (e.g. foods that are liquid at room temperature) [26]. Considering a high proportion of patients in the current study were prescribed oral fluid diets (39\%) may help explain the overall remarkably low provision of nutrition found. This suggests hospitals which have many patients on therapeutic diets, particularly oral fluid diets, are more likely to see higher proportions of patients at risk of PEM.

Approximately a third of patients received sufficient food to meet individual energy and protein requirements. Contrastingly, Thibault et al. [8] and Dupertuis et al. [10] found most patients on full and therapeutic diets received adequate energy and protein to meet their estimated requirements. Whilst therapeutic diets may offer limited dietary choices, it is 
important to consider that they are commonly prescribed following surgery (e.g. oral fluids, TMD) or during acute illness (e.g. low fibre, TMD), when nutritional needs are higher [18]. Therefore, the inadequate provision of nutrition in relation to estimated requirements may, in part, be attributed to higher nutritional needs (due to treatment/illness). Results from the current study indicate this was particularly evident among patients prescribed oral fluid diets, who had the greatest requirements (9497kJ and 93g), yet were provided the least nutrition (3497kJ and 25g). Considering oral fluid diets may be prescribed for prolonged periods of time ( $\geq 3$ days), and are often preceded by periods of fasting [28], these patients are at particular risk of developing profound nutritional deficits. The prescription of oral fluids is likely to contribute to the high prevalence of malnutrition among surgical patients and exacerbate the catabolic response following surgery [29]. Therefore, to reduce the risk and prevalence of PEM, corrective actions are required to improve nutrition delivery among patients prescribed therapeutic diets, particularly oral fluids.

When in hospital, it seems apparent that patients eat significantly less energy and protein than that provided $[8,10]$. Present results suggest other reasons exist for nutritional inadequacy among patients prescribed therapeutic diets in addition to inadequate provision. For example, the majority of patients on TMD were provided adequate energy (80\%) and protein (60\%) to meet their requirements, however, only a minority consumed enough to meet their EER (30\%) and EPR (23\%). Similarly, a high majority of patients on low fibre diets were provided with adequate energy (83\%) and protein (72\%), yet approximately half consumed enough food to meet their EER and EPR. Foodservice satisfaction factors such as the unpleasant taste and appearance of meals, lack of menu choice, and early meal service have been reported as contributing to poor intake, particularly among patients receiving TMD [8, 10, 30]. Of course, disease-related (i.e. severity and type of disease) and treatment-related factors (i.e. poor appetite and nutrition impacting symptoms) may have also contributed to poor intakes, considering therapeutic diets are often prescribed during acute illness/following surgery [9, 13, 15, 31, 32]. Thus, foodservice and patient-related factors may result in suboptimal nutrition intakes. Accommodating for patient preferences, and promptly and effectively managing patients' underlying clinical condition, may be necessary to improve intakes among patients on therapeutic diets.

Given the factors impacting on PEM risk, strategies to combat poor oral intake in patients receiving therapeutic diets need to consider targeting foodservice, nutrition care and patient- 
related issues. Previous studies highlight ONS use can substantially improve nutrition intakes and clinical outcomes among acute care patients [33]. Considering only a quarter of patients prescribed oral fluid diets received ONS ( $\geq 1$ day), routine provision of ONS may offer an immediate practical solution. Further research to assess the clinical and cost-effectiveness of ONS use among patients prescribed therapeutic diets, particularly oral fluid diets, is warranted. In addition, therapeutic diets (particularly oral fluid diets) should only be prescribed when clinically indicated (i.e. for diseases/medical conditions) and not used indiscriminately, given their clear nutritional inadequacy. For example, oral fluid diets (and nil by mouth orders) are unjustified following most surgeries and during acute illness, yet, their high prevalence and unnecessary length of prescription continues to prevail [28, 34, 35]. Understanding why patients are prescribed therapeutic diets in hospital in attempt to inform targeted interventions to reduce their prescription are required. Lastly, patient-related factors (e.g. food preferences and perspectives) should be considered in any intervention targeted at improving nutritional intake. Thus, evaluating patient satisfaction (i.e. through quantitative surveys) and exploring patient perceptions (i.e. through qualitative interviews) may be important to identify if improvements need be made from a foodservice and/or nutritional care perspective, to increase food intake among patients prescribed therapeutic diets (in particular TMD).

We recognise several limitations in our study. Static equations were used to estimate nutritional requirements, consistent with clinical practice; however these equations may have resulted in over or underestimation of some patients' actual nutritional requirements. While the use of indirect calorimetry could be more accurate, resource limitations meant this was not possible. In addition, the accuracy of observed food intake was dependent on the correct serving size of food provided by the kitchen. Whilst foodservice staff at the hospital received training in meal plating and portion sizes, there was potential for variation between staff and shifts. Weighed food intakes are considered the most accurate method of measuring intake, however this was not feasible in the hospital setting and visual observation has been shown to correlate well with weighed intakes [23]. Given the food allergy and intolerance group is represented by small sample size ( $n=6$ patients) further investigation of protein and energy requirements, provisions and intakes are required before findings can be generalised for this dietary group. Lastly, considering a high proportion of our cohort were surgical patients and intake was only measured for 24 hours per patient, we recognise our findings may only be 
applicable to patients with high nutritional requirements and severe nutritional impacting symptoms.

\section{Conclusion}

Energy and protein provisions and intakes are inadequate to meet requirements of all patients prescribed therapeutic diets. Our findings show remarkably lower intakes in proportion to requirements than previous studies conducted among patients on regular diets, suggesting patients prescribed therapeutic diets may be more vulnerable to malnutrition and its associated consequences. Dietary management should therefore be prioritised for patients prescribed therapeutic diets and further research carried out to determine the need for therapeutic diets, and effective strategies to increase dietary intake and reduce the risk of malnutrition among this vulnerable patient population.

\section{Acknowledgments}

We would like to acknowledge Annabel Larby for her assistance with data collection training.

\section{Conflict of interest and funding disclosure}

The authors declare no conflicts of interests. This study received internal institutional support only. 


\section{References}

1. Banks, M., et al., Malnutrition and pressure ulcer risk in adults in Australian health care facilities. Nutrition, 2010. 26(9): p. 896-901.

2. Visvanathan, R., R. Penhall, and I. Chapman, Nutritional screening of older people in a subacute care facility in Australia and its relation to discharge outcomes. Age Ageing, 2004. 33(3): p. 260-5.

3. Isabel, T.D., M. Correia, and D.L. Waitzberg, The impact of malnutrition on morbidity, mortality, length of hospital stay and costs evaluated through a multivariate model analysis. Clin Nutr, 2003. 22(3): p. 235-9.

4. Lim, S., et al., Malnutrition and its impact on cost of hospitalization, length of stay, readmission and 3-year mortality. Clin Nutr, 2012. 31(3): p. 345.

5. Agarwal, E., et al., Malnutrition and poor food intake are associated with prolonged hospital stay, frequent readmissions, and greater in-hospital mortality: results from the Nutrition Care Day Survey 2010. Clin Nutr, 2013. 32(5): p. 737.

6. Stratton, R., C. Green, and M. Elia, Disease-related malnutrition: an evidence-based approach to treatment. 2003, Wallingford, UK: CAB International: p. 93-112.

7. Agarwal, E., et al., Nutritional status and dietary intake of acute care patients: Results from the Nutrition Care Day Survey 2010. Clin Nutr, 2012. 31(1): p. 41-47.

8. Thibault, R., et al., Assessment of food intake in hospitalised patients: A 10-year comparative study of a prospective hospital survey. Clin Nutr, 2011. 30(3): p. 289-296.

9. Hiesmayr, M., et al., Decreased food intake is a risk factor for mortality in hospitalised patients: the NutritionDay survey 2006. Clin Nutr, 2009. 28(5): p. 484-91.

10. Dupertuis, Y.M., et al., Food intake in 1707 hospitalised patients: a prospective comprehensive hospital survey. Clin Nutr, 2003. 22(2): p. 115-123.

11. Bauer, J., et al., nutritionDay: An Australian hospital's participation in international benchmarking on malnutrition. Nutr Diet, 2011. 68(2): p. 134-139.

12. Kandiah, J., L. Stinnett, and D. Lutton, Visual Plate Waste in Hospitalized Patients: Length of Stay and Diet Order. J Am Diet Assoc, 2006. 106(10): p. 1663-1666.

13. Kondrup, J., et al., Incidence of nutritional risk and causes of inadequate nutritional care in hospitals. Clin Nutr, 2002. 21(6): p. 461-468.

14. McLymont, V., S. Cox, and F. Stell, Improving patient meal satisfaction with room service meal delivery. J Nurs Care Qual, 2003. 18(1): p. 27-37.

15. Mudge, A.M., et al., Helping understand nutritional gaps in the elderly (HUNGER): A prospective study of patient factors associated with inadequate nutritional intake in older medical inpatients. Clin Nutr, 2011. 30(3): p. 320-325.

16. Kubrak, C. and L. Jensen, Malnutrition in acute care patients: A narrative review. Int J Nurs Stud, 2007. 44(6): p. 1036-1054.

17. Ray, S., C. Laur, and R. Golubic, Malnutrition in healthcare institutions: A review of the prevalence of under-nutrition in hospitals and care homes since 1994 in England. Clin Nutr, 2014. 33(5): p. 829-835.

18. Norman, K., et al., Prognostic impact of disease-related malnutrition. Clin Nutr, 2008. 27(1): p. 5-15.

19. Niedert, K.C. and A. American Dietetic, Position of the American Dietetic Association: Liberalization of the Diet Prescription Improves Quality of Life for Older Adults in Long-Term Care. J Am Diet Assoc, 2005. 105(12): p. 1955-1965.

20. Larby, A., S. Roberts, and B. Desbrow, Accuracy and adequacy of food supplied in therapeutic diets to hospitalised patients: An observational study. Nutr Diet, 2016: p. n/a-n/a.

21. Wright, L., et al., Comparison of energy and protein intakes of older people consuming a texture modified diet with a normal hospital diet. JHND, 2005. 18(3): p. 213-9. 
22. Queensland Government. Estimating energy, protein \& fluid requirements for adult clinical conditions 2012 [cited 2015 Feb 2]; Available from:

http://www.health.qld.gov.au/nutrition/resources/est rqts.pdf.

23. Olin, A.Ö., et al., Energy-enriched hospital food to improve energy intake in elderly patients. JPEN 1996. 20(2): p. 93-97.

24. Bourdel-Marchasson, I., et al., A multi-center trial of the effects of oral nutritional supplementation in critically ill older inpatients. Nutrition 2000. 16(1): p. 1.

25. Barton, A.D., et al., High food wastage and low nutritional intakes in hospital patients. Clin Nutr, 2000. 19(6): p. 445-449.

26. Hancock, S., G. Cresci, and R. Martindale, The clear liquid diet: When is it appropriate? Curr Gastroenterol Rep, 2002. 4(4): p. 324-331.

27. Shepherd, S.J. and P.R. Gibson, Nutritional inadequacies of the gluten-free diet in both recently-diagnosed and long-term patients with coeliac disease. JHND, 2013. 26(4): p. 349358.

28. Franklin, G.A., et al., Physician-delivered malnutrition: why do patients receive nothing by mouth or a clear liquid diet in a university hospital setting? JPEN, 2011. 35(3): p. 337-42.

29. Ben-Ishay, O., et al., Malnutrition in Surgical Wards: A Plea for Concern. Gastroenterol Res Pract, 2011. 2011: p. 4.

30. Dahl, W.J., S.J. Whiting, and R.T. Tyler, Protein content of puréed diets: Implications for planning. Can J Diet Pract Res, 2007. 68(2): p. 99-102.

31. Patel, M.D. and F.C. Martin, Why don't elderly hospital inpatients eat adequately? J Nutr Health Aging, 2008. 12(4): p. 227-231.

32. Naithani, S., et al., Hospital inpatients' experiences of access to food: a qualitative interview and observational study. Health Expect 2008. 11(3): p. 294-303.

33. Stratton, R.J. and M. Elia, A review of reviews: A new look at the evidence for oral nutritional supplements in clinical practice. Clin Nutr, 2007. 2(1): p. 5-23.

34. Rohatiner, T., et al., A prospective single-institution evaluation of current practices of early postoperative feeding after elective intestinal surgery. Am Surg 2012. 78(10): p. 1147-1150.

35. Lamb, S., et al., 'Nil By Mouth' - Are we starving our patients? e-SPEN, 2010. 5(2): p. e90e92. 